Voix et Images

voixetimages

\title{
Le beau rêve biographique
}

\section{Robert Dion}

Volume 30, numéro 3 (90), printemps 2005

La littérature anglo-québécoise

URI : https://id.erudit.org/iderudit/011867ar

DOI : https://doi.org/10.7202/011867ar

Aller au sommaire du numéro

\section{Éditeur(s)}

Université du Québec à Montréal

\section{ISSN}

0318-9201 (imprimé)

1705-933X (numérique)

Découvrir la revue

Citer ce compte rendu

Dion, R. (2005). Compte rendu de [Le beau rêve biographique]. Voix et Images, 30(3), 183-185. https://doi.org/10.7202/011867ar d'utilisation que vous pouvez consulter en ligne.

https://apropos.erudit.org/fr/usagers/politique-dutilisation/ 


\author{
R E C H E R C H E \\ Le beau rêve biographique \\ $+++$ \\ ROBERT DION \\ Université du Québec à Montréal
}

\begin{abstract}
Alors que paraissait dans Voix et Images le dossier, préparé sous ma direction, consacré aux Avatars du biographique (n 89, hiver 2005), je recevais la douzième livraison des «Archives des lettres canadiennes» portant sur les Approches de la biographie au Québec ${ }^{1}$. Faut-il voir là une heureuse coïncidence ou plutôt l'émergence d'un intérêt soutenu pour le genre, voire le début de la réhabilitation universitaire d'une pratique décriée? Certains indices m'incitent à pencher pour le deuxième terme de l'alternative. Le travail suivi de quelques chercheurs chevronnés (Bernard Andrès, Marcel Olscamp, Lucie Robert, Frances Fortier, notamment) donne en effet à penser que quelque chose bouge, et vite, dans ce secteur jusque-là injustement délaissé. Il faut dire que la production québécoise, plus abondante et plus variée que jamais, a de quoi susciter la curiosité de la critique, qui ne peut plus simplement la refouler dans les marges de l'hagiographie ou du voyeurisme mercantile ${ }^{2}$. Il existe maintenant de solides biographies de quelques-unes de nos figures littéraires, artistiques et politiques majeures, et certaines peuvent même prétendre au statut de littérature - si bien qu'il devenait opportun, pour une série à visée synthétique et récapitulative telle que les "Archives des lettres canadiennes», de consacrer un recueil à ce vaste pan de la production actuelle.
\end{abstract}

Deux cent deux pages, c'est évidemment assez court pour un tour d'horizon de la biographie au Québec. Si Lucie Robert s'acquitte à elle seule très bien de la dimension historique, montrant comment, de 1840 à aujourd'hui, on passe des "vies», hagiographiques et légendaires, aux biographies modernes, et poussant même du côté de ce qu'elle appelle des «hypobiographies» ou "biographies hypothétiques», on pourra déplorer, pour ce qui est de la période contemporaine surtout, d'importantes lacunes, qui tiennent au caractère largement monographique

$$
+++
$$

1 Dominique Lafon, Rainier Grutman, Marcel Olscamp et Robert Vigneault (dir.), Approches de la biographie au Québec, Montréal, Fides, coll. "Archives des lettres canadiennes», 2004, 202 p. 2 Un article de Francine Bordeleau («Biographie: le retour du sujet-roi», Lettres québécoises, n 105, 2002, p. 13-16) témoigne d'ailleurs de la visibilité du phénomène biographique à l'extérieur des cercles très spécialisés. 
de la plupart des contributions du volume. Heureuses exceptions : l'étude de John Hare, qui s'attarde à la sédimentation d'une mémoire biographique autour des leaders patriotes, et celle de Patricia Smart, qui pose la question de la biographie au féminin - qui biographier? selon quel point de vue? dans quel but? - à partir de son livre sur les femmes du Refus global, quoiqu'en envisageant aussi d'autres cas de figure. Ces contributions synthétiques permettent de dégager une vue d'ensemble de quelques secteurs du corpus, vue d'ensemble qu'on aurait souhaité voir s'étendre à la totalité de la «nébuleuse biographique». Elles soulèvent en outre le problème passionnant de la biographie en série et du portrait de groupe, sousgenres qui paraissent devoir convenir aussi bien aux vaincus de l'histoire qu'aux femmes des époques passées.

De construction assez simple, le livre est divisé en deux grandes parties. La première présente «un historique du genre au Québec, des aperçus sur la nature et les formes du genre ainsi que quelques analyses de réalisations exemplaires» (9); la seconde nous fait pénétrer dans l'atelier du biographe: Bernard Andrès, Hélène Pelletier-Baillargeon, Stéphane-Albert Boulais et François Ricard ont charge de nous entraîner dans les coulisses du métier. Quelques «Orientations bibliographiques», trop succinctes pour être vraiment utiles, complètent le recueil.

Évoquant en ouverture du recueil le «beau rêve biographique» dont j'ai fait le titre de ma chronique, et qui est de «raconter une vie» - toute une vie si c'est possible -, Robert Vigneault campe rapidement les grands enjeux et thèmes de l'écriture biographique: art du récit, empathie, fictionnalisation du biographé, fonctions de l'imagination et de l'interprétation. Ces quelques balises étant indiquées, les contributions subséquentes vont sans cesse repasser par là, moduler ces thèmes et enjeux, dans une unanimité au fond assez étonnante: personne, en particulier dans la seconde section du livre sur la pratique biographique, ne semble vouloir se dégager du modèle de la biographie respectueuse, qui fait un usage mesuré de l'imagination et de la fiction, et qui reste fidèle aux faits et aux événements. À l'exception notable de Stéphane-Albert Boulais, qui demande à l'entreprise biographique de l'acheminer vers la connaissance de soi et qui réussit surtout à étaler sa naïve fatuité, les biographes appelés à témoigner, même quand ils disent avoir cherché à concevoir une «biographie romancée» (Andrès), se signalent par leur circonspection et leur sérieux. Ainsi le caractère "romancé» de la biographie que Bernard Andrès consacre à Pierre de Sales Laterrière ne tient-il pas à une fictionnalisation débridée, tant s'en faut, mais à l'importance forcément accordée, dans le cas d'un personnage historique dont l'existence reste pour une bonne part énigmatique, à l'«invention» au sens rhétorique du terme, qui consiste, nous rappelle le biographe, «à rechercher des idées, des sources nouvelles ou non encore exploitées qui garantiront l'originalité du travail» (132).

Les études de cas contemporains, je l'ai mentionné, occupent une part non négligeable de ce tome des "Archives de lettres canadiennes». Vigneault, par exemple, reprend la plume pour aborder le travail biographique et autobiographique de Fernand Ouellette. Chez ce dernier, qui avoue s'identifier totalement à ses modèles (Varèse, Thérèse de Lisieux), la biographie tiendrait davantage de l'essai, qui met au premier plan l'énonciateur et son rapport intime à l'Autre, que du récit, 
qui relate une destinée. Pour Vigneault, est pleinement essayistique (et, partant, littéraire) le texte qui, se rapportant à un élément extérieur - personne admirée, œuvre géniale, etc. - , ne cherche pourtant pas autre chose «que l'élucidation du moi de l'énonciateur» (45). C'est pourquoi l'entreprise biographique, chez Ouellette, n'est pas rigoureusement séparable de l'œuvre autobiographique ni d'ailleurs de l'œuvre critique, puisque, nous dit Vigneault, dans les trois cas il ne s'agit pas tant de comprendre que de co-naître au sens de Claudel, d'une "connaissance empreinte d'amour» (65), d'une intelligence du cœur «entraînant une prise en charge entièrement personnalisée» (47) de l'objet. Pas de théorie biographique ici, par conséquent, mais une valorisation quasi militante de l'identification idiosyncrasique.

Marcel Olscamp se penche pour sa part sur les «intermittences de la biographie» dans l'œuvre de Victor-Lévy Beaulieu. Au principe de son étude, on trouve l'idée, séduisante, d'un «clignotement» (76), d'une traversée du biographique par l'œuvre et de l'œuvre par la biographie, d'une alternance rapide entre visées altéroet autobiographique. Il me semble cependant que la minimisation délibérée du rôle de la biographie chez Beaulieu - celle-ci, d'après Olscamp, n'aurait jamais été chez lui «qu'un phénomène accidentel» (67) - découle d'une conception restrictive du genre. Je préfère, et de beaucoup, la lecture de Jean Morency, qui affirme au contraire que l'écriture biographique "constitue une part importante de [l']entreprise littéraire ${ }^{3}$ » de Beaulieu et qui fait de la dialectique entre essais biographiques et romans autobiographiques l'un des fondements de l'œuvre 4 .

Un mot en terminant sur les témoignages d'Hélène Pelletier-Baillargeon et de François Ricard. La première, dont la compétence en matière d'écriture biographique n'est plus à démontrer, livre un véritable vade mecum à l'usage du biographe débutant. L'humilité, le sérieux et l'expérience parlent ici d'une même voix. Quant au second, il nous livre, entre autres, un intéressant récit de conversion: à l'écriture classique et «maternelle» (l'épithète est de lui) de Gabrielle Roy d'abord, puis à un genre, la biographie, qui avait pour le moins mauvaise presse au sein du milieu universitaire où Ricard a été formé et où il a œuvré par la suite. Nous assistons donc à la genèse du désir biographique et à son explicitation; puis Ricard fait part de sa «morale» du genre - il préfère ce mot à «théorie» - et des principes de sa pratique: ménager à la fois les faits et la pensée, tenir la vie et l'œuvre "à égalité» (191), alterner narration et essai, et ainsi de suite. Dans une contribution qui va bien au delà du seul témoignage, Ricard parvient à exposer les linéaments d'une conception de la biographie d'écrivain et d'une vision de l'œuvre de Roy en relation avec les circonstances de son existence. Ce n'est pas rien, et cela clôt de belle façon un recueil certainement utile.

$$
+++
$$

3 Jean Morency, «Le golem de la biographie. Écriture biographique et écriture de fiction chez Victor-Lévy Beaulieu», Voix et Images, n 89, 2005, p. 22. 4 Il montre par exemple comment l'œuvre romanesque, dans son premier versant, conduit à Monsieur Melville, et comment, dans son second versant, elle se nourrit de la grande trilogie consacrée à l'auteur de Moby Dick. 